\title{
Spectral dimensions from the spectral action
}

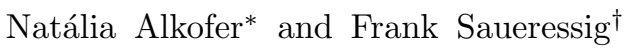 \\ Institute for Mathematics, Astrophysics, and Particle Physics (IMAPP), Radboud University Nijmegen, \\ Heyendaalseweg 135, 6525 AJ Nijmegen, The Netherlands \\ Omar Zanussd \\ Theoretisch-Physikalisches Institut, Friedrich-Schiller-Universität Jena, Max-Wien-Platz 1, 07r43 Jena, Germany
}

(Dated: June 7, 2021)

\begin{abstract}
The generalised spectral dimension $D_{S}(T)$ provides a powerful tool for comparing different approaches to quantum gravity. In this work, we apply this formalism to the classical spectral actions obtained within the framework of almost-commutative geometry. Analysing the propagation of spin-0, spin-1 and spin-2 fields, we show that a non-trivial spectral dimension arises already at the classical level. The effective field theory interpretation of the spectral action yields plateaustructures interpolating between a fixed spin-independent $D_{S}(T)=d_{S}$ for short and $D_{S}(T)=4$ for long diffusion times $T$. Going beyond effective field theory the spectral dimension is completely dominated by the high-momentum properties of the spectral action, yielding $D_{S}(T)=0$ for all spins. Our results support earlier claims that high-energy bosons do not propagate.
\end{abstract}

\section{INTRODUCTION}

The spectral action principle [1, 2] provides a framework for unifying gravity and elementary particle physics on the basis of non-commutative geometry [3], see [4 7 ] for reviews. A key ingredient in the construction is the spectral action [1, 2]

$$
S_{\chi, \Lambda}=\operatorname{Tr}\left[\chi\left(\mathcal{D}^{2} / \Lambda^{2}\right)\right]
$$

where $\chi$ is a positive function, $\mathcal{D}$ is a Dirac operator on a non-commutative geometry, $\Lambda$ is a suitable cutoff scale, and the trace indicates the sum over eigenvalues of $\mathcal{D}$. Amongst non-commutative geometries almostcommutative ones lead, for suitable choices of the almostcommutative manifold, to a spectral action which can give rise to the standard model of particle physics minimally coupled to gravity [8]12. These models may also be extended to include physics beyond the standard model [13 16] or supersymmetry [17 20. Their renormalization has been studied in [21 25] and the phenomenological implications of the resulting effective actions have been carried out, e.g., in [26 28. A more detailed discussion of the cutoff $\Lambda$ may be found in [29], and the generalization to non-commutative spaces built from non-associative algebras has been pursued in [30.

In this work, we follow up on Refs. 31, 32 and study the properties of the spectral action beyond the framework of effective field theory. More specifically, we construct the generalized spectral dimension $D_{S}(T)$ 33 35 . resulting from the spectral action principle and compare our findings with other approaches to quantum gravity.

The basic idea underlying the concept of the generalized spectral dimension $D_{S}(T)$ is that a test particle

\footnotetext{
* N.Alkofer@science.ru.nl

$\dagger$ F.Saueressig@science.ru.nl

$\ddagger$ omar.zanusso@uni-jena.de
}

diffusing on a given background probes certain features of the background. By now, this "observable" has been computed in many approaches to quantum gravity and a number of quantum gravity inspired models. A common feature shared by many of these studies is a "dynamical dimensional reduction" from a classical spacetime with $D_{S}(T)=4$ at macroscopic scales to $D_{S}(T)=2$ [36, 37]. The widespread use of $D_{S}(T)$ throughout the quantum gravity community then calls for a detailed understanding which (quantum) features of a model are actually encoded in $D_{S}(T)$.

In the literature, there are essentially three approaches to compute $D_{S}(T)$. For the piecewise linear geometries approximating spacetime within Monte Carlo simulations of the gravitational partition sum, one sets up a random walk on the effective quantum geometry. This allows a direct measurement of $D_{S}(T)$ from the return probability of the random walker, thereby characterizing the fractal features of the geometry. A prototypical example for this setup is provided by the Causal Dynamical Triangulations (CDT) program 34, 38. The second way towards obtaining a non-trivial $D_{S}(T)$ starts from a theory where the propagator of the test particle is already modified at the classical level. It is this feature that gives rise to the non-trivial spectral dimension in Hořava-Lifshitz gravity [35, 39, 40, general Lorentz-violating theories [41, 42, or fractional quantum field theory [43, 44]. Finally, $D_{S}(T)$ may be modified through non-trivial quantum fluctuations of spacetime predicted by a fundamental theory of gravity. This set includes the multifractal spacetimes occurring in the gravitational Asymptotic Safety program [33, 45] 48, the microscopic structure of spacetime within Loop Quantum Gravity [4954, Causal Set Theory [55] or the propagation of particles on $\kappa$-Minkowski [56, 57] and other non-commutative spaces [58].

For the classical spectral action, the non-trivial spectral dimension originates from the non-canonical momentum dependence of the theory's propagators. The essentially new feature, making this model worthwhile to 
study, is that the (inverse) full propagators of the model are non-analytic functions of the momentum. This will lead to a rather surprising behaviour of the spectral dimension computed in this framework.

The rest of this work is devoted towards studying these novel features. In Sects. II and III we review the relevant aspects of the spectral action and the generalized spectral dimension, respectively. The spectral dimension resulting from the spectral action is computed in Sect. IV and we conclude with a brief summary and outlook in Sect. V.

\section{THE SPECTRAL ACTION AND ITS BOSONIC PROPAGATORS}

We start by reviewing the spectral action and its connection to the heat-kernel mainly following [7, 31]. The basic ingredient for constructing the spectral action is an almost-commutative manifold $M \times F$. Here $M$ is a Riemannian spin manifold which plays the role of the Euclidean spacetime and $F$ a finite, generally noncommutative, space encoding the internal degrees of freedom. The geometry of $M$ has a operator algebraic description in terms of the canonical triple

$$
M:=\left(C^{\infty}(M), L^{2}(M, S), \mathcal{D}\right)
$$

where $C^{\infty}(M)$ is the set of smooth functions on $M$, $L^{2}(M, S)$ is the Hilbert space of square-integrable spinors on $M$, and $\mathcal{D}$ is the (Euclidean) Dirac operator acting on this Hilbert space. Similarly, the geometry of $F$ can be captured by a triple

$$
F:=\left(\mathcal{A}_{F}, \mathcal{H}_{F}, D_{F}\right)
$$

where $\mathcal{H}_{F}$ is a finite-dimensional Hilbert space of complex dimension $N, \mathcal{A}_{F}$ is an algebra of $N \times N$ matrices acting on $\mathcal{H}_{F}$, and $D_{F}$ is a hermitian operator, given by a hermitian $N \times N$ matrix.

In order to illustrate the propagation of particles resulting from this setup, it suffices to chose the simplest internal space, taking $F$ as a single point. In this case the triple $F=(\mathbb{C}, \mathbb{C}, 0)$ and the Dirac operator on the product space reduces to the one on $M$. Concretely, we will consider

$$
\mathcal{D}=\not D+\gamma_{5} \phi
$$

where the covariant derivative

$$
\not D=i \gamma^{\mu}\left(\nabla_{\mu}^{L C}+i A_{\mu}\right)
$$

contains the Levi-Cevita spin connection and the gauge potential $A_{\mu}$. Consequently, the resulting spectral action comprises a spin-2 field, the graviton, a massless $U(1)$ gauge field $A_{\mu}$ with field strength $F_{\mu \nu}=\partial_{\mu} A_{\nu}-\partial_{\nu} A_{\mu}$ and a scalar $\phi$. In the sequel, we will study the transverse traceless fluctuations $h_{\mu \nu}$ with $\partial^{\mu} h_{\mu \nu}=0, \delta^{\mu \nu} h_{\mu \nu}=0$ only and impose the Landau gauge for the spin-1 field $\partial^{\mu} A_{\mu}=0$, limiting ourselves to the propagation of the physical degrees of freedom.

The operator $\mathcal{D}^{2}$ appearing in the spectral action (1) can then be cast into the standard form of a Laplace-type operator

$$
\mathcal{D}^{2}=-\left(\nabla^{2}+E\right)
$$

with the endomorphism $E$ given by

$$
E=-i \gamma^{\mu} \gamma_{5} \nabla_{\mu} \phi-\phi^{2}-\frac{1}{4} R+\frac{i}{4}\left[\gamma^{\mu}, \gamma^{\nu}\right] F_{\mu \nu}
$$

Moreover, the curvature of $\nabla_{\mu}=\nabla_{\mu}^{L C}+i A_{\mu}$ is given by

$$
\Omega_{\mu \nu}:=\left[\nabla_{\mu}, \nabla_{\nu}\right]=-\frac{1}{4} \gamma^{\rho} \gamma^{\sigma} R_{\rho \sigma \mu \nu}+i F_{\mu \nu} .
$$

At this stage, it is illustrative to follow 31 and consider the special case where the generating function in eq. (1) is given by $\chi(z)=e^{-z}$ In this case, the spectral action (1) coincides with the heat trace

$$
S_{\chi, \Lambda}=\operatorname{Tr}\left(e^{-t \mathcal{D}^{2}}\right)=K\left(\mathcal{D}^{2}, t\right) \quad \text { with } \quad t:=\Lambda^{-2}
$$

which is a well-studied object, see, e.g., [32, 60 63.

As we are interested in the propagation of fields we will extract the (inverse) propagators of the matter fields by expanding (9) up to second order in $\phi, A_{\mu}$. The inverse propagator in the gravity sector is obtained by expanding $g_{\mu \nu}$ around flat (Euclidean) space

$$
g_{\mu \nu}=\delta_{\mu \nu}+\Lambda^{-1} h_{\mu \nu} .
$$

The inclusion of $\Lambda$ ensures that $h_{\mu \nu}$ has the same massdimension as the matter fields and gives rise to the canonical form of the graviton propagator. Comparing (10) with the stand expansion of $g_{\mu \nu}$ used in perturbation theory, $g_{\mu \nu}=\delta_{\mu \nu}+\sqrt{16 \pi G_{\mathrm{N}}} h_{\mu \nu}$ with $G_{\mathrm{N}}$ being Newton's constant, identifies the natural scale for $\Lambda$ as the Planck mass $m_{\mathrm{Pl}}=\left(8 \pi G_{\mathrm{N}}\right)^{-1 / 2}$ (also see 64 for a related discussion). A lengthy but in principle straightforward calculation [31, 32, 60, 62 then yields the expression for the inverse propagators of the physical fields including the full-momentum dependence

$$
\begin{aligned}
& K^{(2)}\left(\mathcal{D}^{2}, t\right)=\int d^{4} x\left[\phi F_{0}\left(-t \partial^{2}\right) \phi\right. \\
& \left.\quad+A_{\mu} F_{1}\left(-t \partial^{2}\right) A_{\mu}+\Lambda^{-2} h_{\mu \nu} F_{2}\left(-t \partial^{2}\right) h_{\mu \nu}\right]
\end{aligned}
$$

Here $K^{(2)}$ indicates that we retained the second order of the fields only and we explicitly exhibit the factor $\Lambda^{-2}$ coming from the expansion (10). The structure functions $F_{s}$ coincide with the standard heat kernel result for spin- $s$ fields and are given by

$$
\begin{aligned}
& F_{0}(z)=\frac{t^{-1}}{(4 \pi)^{2}}(-4+2 z h(z)), \\
& F_{1}(z)=\frac{t^{-1}}{(4 \pi)^{2}}(-4+4 h(z)+2 z h(z)), \\
& F_{2}(z)=\frac{t^{-2}}{(4 \pi)^{2}}\left(-2+h(z)+\frac{1}{4} z h(z)\right),
\end{aligned}
$$




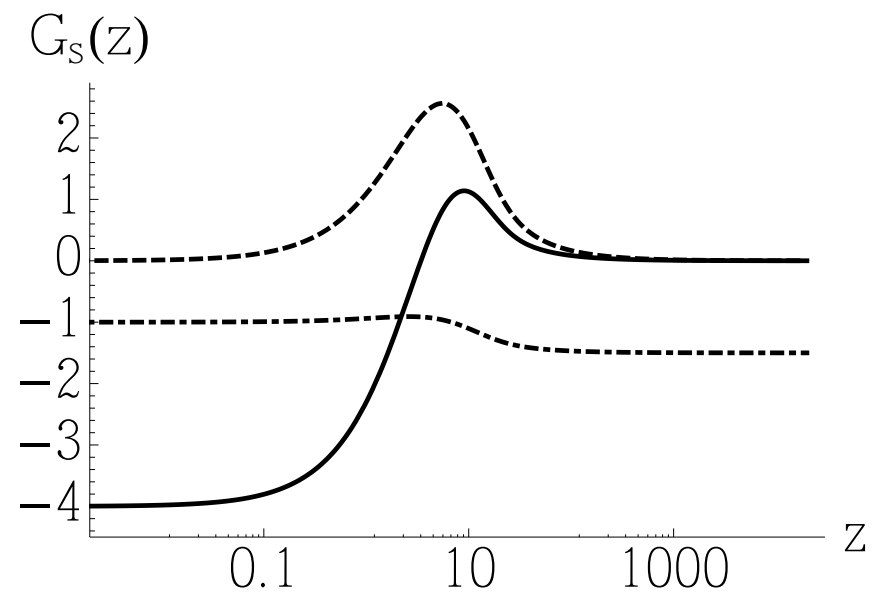

FIG. 1. Illustration of the momentum dependence of the structure functions $G_{s}(z)$ (17): spin 0 - solid thick line, spin 1- dashed thick line, and spin 2 - dash-dotted thick line.

with

$$
h(z)=\int_{0}^{1} d \alpha e^{-\alpha(1-\alpha) z} .
$$

Note that these functions are non-analytic in

$$
z=\frac{p^{2}}{\Lambda^{2}}=t p^{2}
$$

The inverse of these structure functions provide the classical propagators of the theory. For illustration, we show the "reduced" structure functions

$$
G_{s}(z)=(4 \pi)^{2} t^{\alpha_{s}} F_{s}(z)
$$

with $F_{s}(z)$ defined in eqs. (12) - 14) and $\alpha_{s}=(1,1,2)$ in Fig. 1 .

The structure functions $(12)-(14)$ posses an early-time expansion for momenta $p^{2} \ll \Lambda^{2}$, i.e., $z<1$. Using that

$$
h(z)=1-\frac{z}{6}+\frac{z^{2}}{60}-\frac{z^{3}}{840}+\mathcal{O}\left(z^{4}\right)
$$

one finds

$$
\begin{aligned}
& (4 \pi)^{2} t F_{0}(z)=-4+2 z-\frac{z^{2}}{3}+\frac{z^{3}}{30}+\ldots, \\
& (4 \pi)^{2} t F_{1}(z)=\frac{4}{3} z-\frac{4}{15} z^{2}+\frac{1}{35} z^{3}+\ldots, \\
& (4 \pi t)^{2} F_{2}(z)=-1+\frac{z}{12}-\frac{z^{2}}{40}+\frac{z^{3}}{336}+\ldots
\end{aligned}
$$

The early time expansion of the structure functions (truncated at $\mathcal{O}\left(z^{3}\right)$ and $\mathcal{O}(z)$, respectively) is shown in Fig. 2. Comparing the result to the functions $F_{s}(z)$ including the full $z$-dependence it is easily seen that the truncation drastically modifies the behaviour of the (inverse) propagators for large momenta. While the truncated $F_{s}(z)$ diverge the full structure functions remain

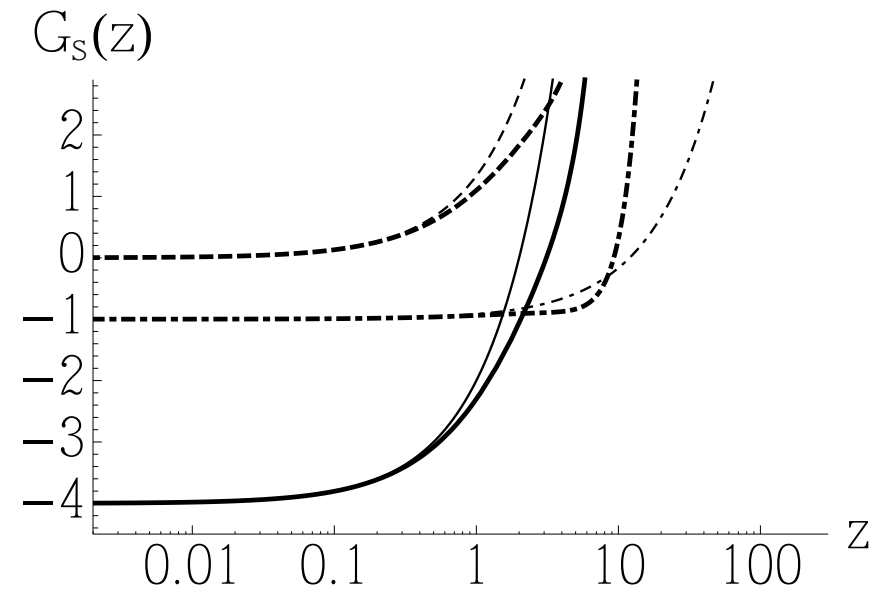

FIG. 2. Propagators obtained from truncating $G_{s}(z)$ at $z^{3}$, following the spirit of effective field theory (spin 0 - solid thick line, spin 1- dashed thick line, and spin 2 - dash-dotted). The expansions up to linear order in $z$ are shown as corresponding thin lines.

finite. As we will see in Sect. IV] this feature will have a drastic effect on the spectral dimension of the theory.

At this stage, the following remarks are in order. The constant term appearing in the expansion of $F_{0}$ plays the role of a mass term for $\phi$. The sign thereby indicates that the squared mass is negative. This is a remnant of the fact that the scalar $\phi$ acquires a non-trivial vacuum expectation value via the Higgs mechanism [1, 11, 24. Since $K^{(2)}$, by construction, contains the terms quadratic in $\phi$ only the scalar potential is not included in (11) so that the stabilisation of $\phi$ cannot be demonstrated in this approximation. The constant term in $F_{2}$ indicates the presence of a positive cosmological constant, acting like a mass-term for the graviton, while the structure of $F_{1}$ indicates that the gauge field remains massless.

In order to understand the behaviour of the theory at high energies, it is also useful to carry out the latetime expansion of the structure functions, capturing the behaviour for $z \gg 1$. In this case

$$
h(z)=\frac{2}{z}+\frac{4}{z^{2}}+\frac{24}{z^{3}}+\frac{240}{z^{4}}+\ldots
$$

which yields

$$
\begin{aligned}
& (4 \pi)^{2} t F_{0}(z)=\frac{8}{z}+\frac{48}{z^{2}}+\frac{480}{z^{3}}+\ldots, \\
& (4 \pi)^{2} t F_{1}(z)=\frac{16}{z}+\frac{64}{z^{2}}+\frac{576}{z^{3}}+\ldots, \\
& (4 \pi t)^{2} F_{2}(z)=-\frac{3}{2}+\frac{3}{z}+\frac{10}{z^{2}}+\frac{84}{z^{3}}+\ldots
\end{aligned}
$$

A typical viewpoint adopted in the spectral action approach to particle physics consider the actions generated by (1) as effective actions which should be truncated at a certain power of the cutoff $\Lambda^{-2}$. In this case the earlytime expansion $(19)$ allows to construct the effective action resulting from an arbitrary function $\chi$. This uses the 
fact that the Trace (1) can be related to the heat kernel (9) using

$$
\begin{aligned}
S_{\chi, \Lambda} & =\operatorname{Tr}\left[\chi\left(\mathcal{D}^{2} / \Lambda^{2}\right)\right] \\
& =\int_{0}^{\infty} d y \tilde{\chi}(y) \operatorname{Tr}\left[e^{-y \mathcal{D}^{2} / \Lambda^{2}}\right],
\end{aligned}
$$

where $\tilde{\chi}(y)$ is the inverse Laplace-transform of $\chi(z)$. Evaluating the operator trace in $(22)$ based on the earlytime expansion then yields the systematic expansion of $S_{\chi, \Lambda}$ in (inverse) powers of the cutoff. The $\chi$-dependent coefficients in this expansion are given by

$$
Q_{n}[\chi] \equiv \int_{0}^{\infty} d y y^{-n} \tilde{\chi}(y)
$$

and can be computed by standard Mellin-transform techniques [59]. Thus the $Q_{n} \equiv Q_{n}[\chi]$ are real numbers which are normalized such that $Q_{n}=1$ for $\chi=\exp (-t z)$.

The part of the spectral action containing the terms quadratic in the fields is then given by

$$
\begin{aligned}
S_{\chi, \Lambda}^{(2)}=\frac{\Lambda^{2}}{(4 \pi)^{2}} \int d^{4} x & {\left[\phi \mathcal{F}_{0, \chi}\left(-\partial^{2} / \Lambda^{2}\right) \phi\right.} \\
& +A_{\mu} \mathcal{F}_{1, \chi}\left(-\partial^{2} / \Lambda^{2}\right) A_{\mu} \\
& \left.+h_{\mu \nu} \mathcal{F}_{2, \chi}\left(-\partial^{2} / \Lambda^{2}\right) h_{\mu \nu}\right] .
\end{aligned}
$$

with

$$
\begin{aligned}
& \mathcal{F}_{0, \chi}(z)=-4 Q_{1}+2 Q_{0} z-\frac{Q_{-1}}{3} z^{2}+\frac{Q_{-2}}{30} z^{3}+\ldots, \\
& \mathcal{F}_{1, \chi}(z)=\frac{4 Q_{0}}{3} z-\frac{4 Q_{-1}}{15} z^{2}+\frac{Q_{-2}}{35} z^{3}+\ldots, \\
& \mathcal{F}_{2, \chi}(z)=-Q_{2}+\frac{Q_{1}}{12} z-\frac{Q_{0}}{40} z^{2}+\frac{Q_{-1}}{336} z^{3}+\ldots
\end{aligned}
$$

The momenta (23) can be adjusted by choosing a suitable function $\chi$. Note, however, that the $Q_{n}$ 's appearing in the matter and gravitational sector of (24) cannot be adjusted independently, however, since they are generated by the same function $\chi$.

We close this section by discussing possible truncations of the expansion (11):

Truncating the moments $Q_{n}$. From the mathematical viewpoint it is tempting to choose a generating function $\chi$ whose moments $Q_{n}[\chi]$ vanish for all values $n \geq n_{\max }$. This leads to the rather peculiar property that the highest powers of $-\partial^{2}$ appearing in the matter and gravitational sector come with opposite signs. In other words adjusting the $Q_{n}$ in such a way that the propagators in the matter sector are stable at high momenta implies an instability of the gravitational propagator and vice versa. Thus "truncating" the theory by adjusting the momenta $Q_{n}$ gives rise to a dynamical instability of the theory.

The effective field theory viewpoint. A similar (though not equivalent) strategy interprets the expansion (11) as an effective field theory, which should be truncated at a given power of the cutoff $\Lambda$. Retaining the relevant and marginal operators then provides a good description of the physics as long as $-p^{2} / \Lambda^{2} \ll 1$. While it is possible to systematically compute quantum corrections to an effective action, this expansion breaks down if the momenta are of the order of the Planck scale. A detailed analysis then reveals that the $Q_{n}$ 's can be adjusted in such a way that all propagators of the theory are stable. Thus we will focus on this case in the sequel.

\section{THE GENERALIZED SPECTRAL DIMENSION}

The motivation for studying the (generalized) spectral dimension associated with a particle physics model comes from the idea that a test particle diffusing on a given fixed background feels certain features of this background as, e.g., its dimension. For a spin-less test-particle performing a Brownian random walk on a Riemannian manifold with metric $g_{\mu \nu}$, the diffusion process is described by the heat kernel $K_{g}\left(x, x^{\prime} ; T\right)$ which gives the probability density for a particle diffusing from the point $x$ to $x^{\prime}$ in the diffusion time $T$. The heat kernel satisfies the heat equation

$$
\begin{aligned}
& \left(\partial_{T}+\Delta_{g}\right) K_{g}\left(x, x^{\prime} ; T\right)=0, \\
& K_{g}\left(x, x^{\prime} ; 0\right)=\delta\left(x-x^{\prime}\right) .
\end{aligned}
$$

where $\Delta_{g} \equiv-D^{2}$ is the Laplace-Beltrami operator. In flat space, the solution of this equation is

$$
K\left(x, x^{\prime} ; T\right)=\int \frac{d^{d} p}{(2 \pi)^{d}} e^{i p \cdot\left(x-x^{\prime}\right)} e^{-p^{2} T} .
$$

In general $K_{g}\left(x, x^{\prime} ; T\right)$ is the matrix element of the operator $\exp \left(-T \Delta_{g}\right)$. For the diffusion process, its trace per volume gives the averaged return probability

$$
\begin{aligned}
\mathcal{P}_{g}(T) & =V^{-1} \int d^{d} x \sqrt{g(x)} K_{g}(x, x ; T) \\
& =V^{-1} \operatorname{Tr} \exp \left(-T \Delta_{g}\right)
\end{aligned}
$$

measuring the probability that the particle returns to its origin after a diffusion time $T$. Here $V \equiv \int d^{d} x \sqrt{g(x)}$ denotes the total volume. For the flat-space solution (27)

$$
\mathcal{P}(T)=(4 \pi T)^{-d / 2} .
$$

The (standard) spectral dimension $d_{S}$ is defined as the $T$-independent logarithmic derivative

$$
d_{S} \equiv-2 \lim _{T \rightarrow 0} \frac{\partial \ln \mathcal{P}(T)}{\partial \ln T} .
$$

On smooth manifolds $d_{S}$ agrees with the topological dimension of the manifold $d$. In order to also capture 
the case of diffusion processes exhibiting multiple scaling regimes, it is natural to generalize the definition 30 to the $T$-dependent spectral dimension

$$
D_{S}(T) \equiv-2 \frac{\partial \ln \mathcal{P}(T)}{\partial \ln T}
$$

In the classical spectral action (1) the propagation of the test particles on a flat Euclidean background is modified by the higher-derivative terms entering into the (inverse) propagators of the fields. In eq. 26 this effect can readily be incorporated by replacing the LaplaceBeltrami operator by the inverse propagators

$$
\begin{aligned}
& \left(\partial_{T}+F\left(-\partial^{2}\right)\right) K_{g}\left(x, x^{\prime} ; T\right)=0, \\
& K_{g}\left(x, x^{\prime} ; 0\right)=\delta\left(x-x^{\prime}\right) .
\end{aligned}
$$

The solution of this equation can again be given in terms of its Fourier transform

$$
K\left(x, x^{\prime} ; T\right)=\int \frac{d^{d} p}{(2 \pi)^{d}} e^{i p \cdot\left(x-x^{\prime}\right)} e^{-F\left(p^{2}\right) T} .
$$

Notably, given a generic function $F\left(p^{2}\right)$ there is no guarantee that the resulting heat-kernel is positive semidefinite thereby admitting an interpretation as probability density. This "negative probability problem" has been discussed in detail [45, 65, concluding that the spectral dimension remains meaningful.

The $\mathcal{P}(T)$ resulting from 33 is given by

$$
\mathcal{P}(T)=\int \frac{d^{d} p}{(2 \pi)^{d}} e^{-F\left(p^{2}\right) T} .
$$

and may still admit the interpretation of a (positivesemidefinite) return probability even in the case where a probability interpretation of $K\left(x, x^{\prime} ; T\right)$ fails. The generalized spectral dimension may then be obtained by substituting the inverse propagators from eq. (11) and evaluating (31) for the corresponding return probabilities.

Following the ideas of [41, 42] the spectral dimension arising from (34) permits an interpretation as the Hausdorff-dimension of the theory's momentum space. Provided that the change of coordinates $k^{2}=F\left(p^{2}\right)$ is bijective, the inverse propagator in the exponential can be traded for a non-trivial measure on momentum space

$$
P(T)=\frac{\mathrm{Vol}_{S^{d}}}{(2 \pi)^{d}} \int k d k \frac{\left(F^{-1}\left(k^{2}\right)\right)^{(d-2) / 2}}{F^{\prime}\left(p^{2}\right)} e^{-T k^{2}} .
$$

Here we invoked the inverse function theorem where $F^{\prime}\left(p^{2}\right)$ is understood as the derivative of $F(z)$ with respect to its argument, evaluated at $p^{2}=F^{-1}\left(k^{2}\right)$. Thus eq. (34) is equivalent to a particle with canonical inverse propagator, $F\left(p^{2}\right) \propto p^{2}$ in a momentum space with nontrivial measure. This picture also provides a meaningful interpretation of $D_{S}(T)$ even in the case where the model is purely classical so that the non-trivial spectral dimension cannot originate from properties of an effective quantum spacetime.
Before embarking on the computation of the spectral dimensions resulting from the spectral action it is illustrative to consider the case where the inverse momentumspace propagator $F\left(p^{2}\right)$ contains a mass term $m^{2}=$ $\left.F\left(p^{2}\right)\right|_{p^{2}=0}$. In this case it is useful to split off the massless part from $F\left(p^{2}\right)$ and write

$$
F\left(p^{2}\right)=F^{(0)}\left(p^{2}\right)+m^{2} .
$$

Based on $F^{(0)}\left(k^{2}\right)$ we can then introduce the return probability

$$
P^{(0)}(T) \propto \int \frac{d^{4} p}{(2 \pi)^{4}} e^{-T F^{(0)}\left(p^{2}\right)}
$$

together with the spectral dimension seen by the massless field

$$
D_{S}^{(0)}(T) \equiv-2 T \frac{\partial}{\partial T} \ln \mathcal{P}^{(0)}(T) .
$$

Substituting 36 into the return probability 34 and extracting the mass-term from the integral it is straightforward to establish

$$
D_{S}(T)=2 m^{2} T+D_{S}^{(0)}(T) .
$$

Thus a mass-term just leads to a linear contribution in $D_{S}(T)$ and does not encode non-trivial information on the propagation of the particle. Thus we limit ourselves to the study of $D_{S}^{(0)}(T)$ in the sequel.

\section{THE SPECTRAL DIMENSION FROM THE SPECTRAL ACTION}

Based on the discussion of the last two sections, it is now straightforward to compute the spectral dimensions from the spin-dependent propagators provided by the spectral action. We will start by investigating the truncated propagators based on eqs. (11) and 24) in Subsection IV A before including the full momentum dependence in Subsection IVB.

\section{A. Effective field theory framework}

In the effective field theory interpretation of $(24)$ the functions $\mathcal{F}_{s, \chi}$ are truncated at a fixed power of the cutoff $\Lambda$. The resulting massless parts of the bosonic propagators then become polynomials in the particles momentum,

$$
\mathcal{F}_{s}^{(0)}\left(p^{2}\right)=\sum_{n=1}^{N_{\max }} a_{n}^{s}\left(p^{2}\right)^{n}
$$

with obvious relations among the polynomial coefficients $a_{n}$ and the numbers $Q_{n}$ 25). Limiting the expansion to the marginal and relevant operators, coming with powers of the cutoff $\Lambda^{2 n}, n \leq 2$, fixes $N_{\max }=1$ and all 


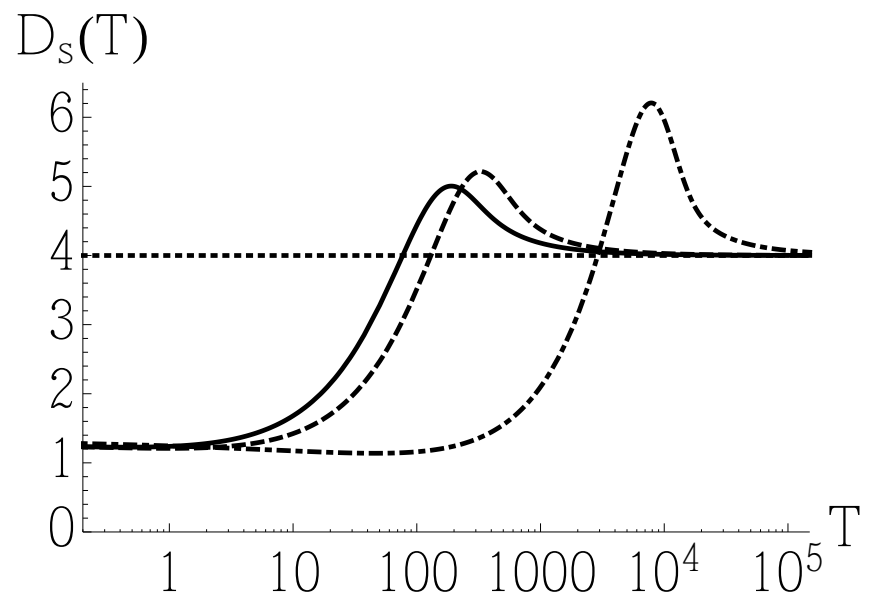

FIG. 3. The spin-dependent spectral dimension $D_{S}^{(0)}(T)$ obtained from 40 with $N_{\max }=1$ (dotted line) and $N_{\max }=$ $3, Q_{n}=1, n=1,0,-1,-2$ for spin 0 - solid line, spin 1dashed line, and spin 2 - dashed-dotted line. The cross-over scale is set by $t=\Lambda^{-2}$ and has been normalized to $t=1$.

propagators retain their standard $p^{2}$-form. Also taking into account power-counting irrelevant terms containing inverse powers of the cutoff adds further powers to the polynomial 40). Thus the propagators include higher powers of momentum in this case.

A positive semi-definite spectral dimension $D_{S}^{(0)}$ requires a positive function $\mathcal{F}_{s}^{(0)}$. This requirement puts constraints on the signs of the momenta $Q_{n}$ appearing in eq. 25). In particular $a_{1}^{s}>0$ is required for obtaining classical propagators at low energies while $a_{N_{\max }}^{s}>0$ is needed for stability at high energies.

The asymptotic behavior of $D_{S}^{(0)}$ for short (long) diffusion time $T$ is governed by the highest (lowest) power of $p^{2}$ contained in (40). Evaluating (37) and (38) for the special cases $\mathcal{F}_{s}^{(0)}\left(p^{2}\right) \propto p^{2}$ and $F\left(p^{2}\right) \propto\left(p^{2}\right)^{N_{\max }}$, a simple scaling argument establishes

$$
\begin{aligned}
& \lim _{T \rightarrow \infty} D_{S}^{(0)}(T)=4, \quad a_{1}>0, \\
& \lim _{T \rightarrow 0} D_{S}^{(0)}(T)=\frac{4}{N_{\max }} .
\end{aligned}
$$

Thus $a_{1}>0$ ensures that the spectral dimension seen by particles for long diffusion times coincides with the topological dimension of spacetime. Including higher powers of momenta decreases $D_{S}^{(0)}(T)$ for short diffusion times. The generalized spectral dimension then interpolates smoothly between these limits. This feature is illustrated in Fig. 3. The case $N_{\max }=1, a_{1}>0$ leads to a spectral dimension which is independent of the diffusion time (dashed line). The spectral dimension obtained for $N_{\max }=3$ and momenta $Q_{n}=1, n=1,0,-1,-2$. In this case, $D_{S}^{(0)}(T)$ interpolates between 4 at large $T$ and $4 / 3$ for small $T$ respectively. The crossover occurs for $T / t \approx(4 \pi)^{2}$ (Spin 0 and 1 ), resp., $T / t^{2} \approx 10 \times(4 \pi)^{2}$ (Spin 2 ). Notably, it is only the shape of this crossover, which depends on the spin of the particle, while the asymptotic limits are universal for all spins.

\section{B. Propagators with full momentum dependence}

We now take the step beyond effective field theory and investigate the spectral dimension arising from the inverse propagators (14) including the full momentum dependence. Comparing Figs. 1 and 2 the structural difference is immediate: in the effective field theory framework $F_{s}(z)$ diverges as $z \rightarrow \infty$ while the inclusion of the full momentum dependence renders $\lim _{z \rightarrow \infty} F_{s}(z)$ finite with the limit given by the leading term in 211). As a consequence of the modified asymptotics, the integral (37) diverges at the upper boundary, since the contribution of large momenta is no longer exponentially suppressed once the full momentum dependent propagators are considered.

In order to still be able to analyse the spectral dimension arising in this framework, we regulate (37) by introducing a UV cutoff $\Lambda_{\mathrm{UV}}$,

$$
P^{(0)}\left(T ; \Lambda_{\mathrm{UV}}\right)=\int^{\Lambda_{\mathrm{UV}}} \frac{d^{4} p}{(2 \pi)^{4}} e^{-T F^{(0)}\left(p^{2}\right)} .
$$

In the spirit of the discussion leading to eq. $(39)$, we consider the "massless" structure functions $F_{s}^{(0)}(z)$ where the constant terms appearing in the late-time expansion (21) have been removed. The return probability 42 then allows to construct the spectral dimension as a function of $\Lambda_{\mathrm{UV}}$

$$
D_{S}^{(0)}\left(T ; \Lambda_{\mathrm{UV}}\right)=-2 T \partial_{T} \ln \left(P^{(0)}\left(T ; \Lambda_{\mathrm{UV}}\right)\right) .
$$

The scale $\Lambda$ appearing in the spectral action (1) is thereby held fixed and sets the transition scale between the UV and IR regime. A detailed analytical and numerical analysis based on the expansion (21) then establishes

$$
\lim _{\Lambda_{\mathrm{UV}} \rightarrow \infty} D_{S}^{(0)}\left(T ; \Lambda_{\mathrm{UV}}\right)=\lim _{\Lambda_{\mathrm{UV}} \rightarrow \infty} 4 T F_{s}^{(0)}\left(\Lambda_{\mathrm{UV}}\right) .
$$

Based on the late-time expansion 21, we thus we conclude that including the full momentum dependence in the structure function leads to a spectral dimension which vanishes for all diffusion times $T$ :

$$
D_{S}^{(0)}(T)=\lim _{\Lambda_{\mathrm{UV}} \rightarrow \infty} D_{S}^{(0)}\left(T ; \Lambda_{\mathrm{UV}}\right)=0 .
$$

This result entails in particular that there is no scaling regime for large diffusion time where the spectral dimension matches the topological dimension. Thus including the full momentum dependence, one does not recover a "classical regime" were the spectral dimension would indicate the onset of classical low-energy physics. 


\section{CONCLUSIONS AND OUTLOOK}

In this work we constructed the generalized spectral dimension $D_{S}(T)$ describing the propagation of (massless) scalars, vectors and gravitons based on the classical spectral action (1). Our results distinguish three cases:

1. If the spectral action is interpreted as an effective field theory restricted to the power-counting relevant and marginal terms, the generalized spectral dimension is independent of the diffusion time $T$ and matches the topological dimension of spacetime $D_{S}(T)=4$.

2. If the effective field theory framework is extended to also include power-counting irrelevant terms, the generalized spectral action interpolates between $D_{S}(T)=4$ for long diffusion time and $D_{S}(T)=$ $4 / N_{\max }$ for short diffusion times. $N_{\max }$ is determined by the highest power of momentum contained in the propagator, $\left(p^{2}\right)^{N_{\max }}$. The crossover between these two asymptotic regimes is set by the cutoff $\Lambda$ and its shape explicitly depends on the spin of the propagating particle.

3. If the full momentum-dependence of the propagators is taken into account, the generalized spectral dimension becomes independent of the spin and vanishes identically $D_{S}(T)=0$.

The last feature can be traced back to the fact that the full propagators approach a constant for momenta much larger than the characteristic cutoff scale $\Lambda$. In Ref. 31] the peculiar behaviour was summarized by the catchy phrase that "high-energy bosons do not propagate". Indeed the vanishing of the spectral dimension suggests that the momentum space of the theory resembles the one of a zero-dimensional field theory. In this sense the situation resembles a picture where spacetime fragments into isolated points which do not communicate.

We stress, however, that all computations carried out in this work are at the classical level. In particular the spacetime is given by classical Euclidean space. All effects are due to the change of measure in momentum space reflected by non-canonical form of the classical propagators and thus do not capture properties of an underlying quantum spacetime. Nevertheless, we believe that the spectral action, comprising the three scenarios discussed above, provides a valuable testbed for new candidates for quantum gravity observables generalizing the spectral dimension.

The vanishing of the spectral dimension, $D_{S}(T)=0$, is in agreement with the previous computations obtained for other non-commutative spacetimes [58]. This is a welcome and surprising result, since the non-commutative nature of our spectral triple construction differs substantially from that of [58]. Aside for the limiting case of vanishing spectral dimension, both results display the same qualitative features: The spectral dimension interpolates between the topological dimension and zero, and has a local maximum situated close to a transition scale. Additionally, the onset of the transition is, in both cases, controlled by the parameter which is introduced by the non-commutative description ( $\Lambda$ in our computation and the parameter $\kappa$ that governs the non-commutativity of the coordinates in [58]). Therefore, the transition is truly representative of a regime in which the non-commutative features of the spacetime become important.

In agreement with the conjecture of "Asymptotic Silence" pushed forward in 36, it is a general feature of our computation that the spectral dimension decreases in the ultraviolet, and thus describes a spacetimes that breaks into disconnected regions at high energies. However, differently from 36, it is not immediately evident what is the physical mechanism underlying this scenario. Furthermore, our resuts are instead in stark contrast to the generalized spectral dimension typically obtained within quantum gravity approaches where $D_{S}(T)$ typically interpolates between $D_{S}(T)=4$ (classical, macroscopic phase) and $D_{S}(T)<4$ at microscopic scales [36].

At this stage, it is tempting to speculate that the vanishing spectral dimension is an artefact of extrapolating the classical spectral action into the trans-Planckian regime without taking quantization effects into account. In other words, vacuum fluctuations seem to be fundamental to balance the fragmentation of spacetime in the ultraviolet [37]. An UV completion of the spectral action could be achieved through the Asymptotic Safety mechanism [47, 59], which seems a natural choice given the field content and symmetries of the model, may lead to $D_{S}(T)$ interpolating from four to two, while still keeping the non-polynomial momentum dependence in the propagators. We hope to come back to this intriguing possibility in the future.

\section{ACKNOWLEDGMENTS}

We thank M.A. Kurkov, Fedele Lizzi and Martin Reuter for inspiring discussions and Walter van Suijlekom for helpful comments and a critical reading of the manuscript. N.A. and F.S. are financially supported by the Netherlands Organization for Scientific Research (NWO) within the Foundation for Fundamental Research on Matter (FOM) grants 13PR3137 and 13VP12. O.Z. acknowledges financially supported by the DFG within the Emmy-Noether program (Grant SA/1975 1-1) while this work was in preparation. 
[1] A. H. Chamseddine and A. Connes, Phys. Rev. Lett. 77 (1996) 4868.

[2] A. H. Chamseddine and A. Connes, Commun. Math. Phys. 186 (1997) 731 hep-th/9606001.

[3] A. Connes, "Noncommutative geometry," Academic Press, San Diego (1994).

[4] A. Connes, Noncommutative geometry and physics, in B. Julia, J. Zinn-Justin (Eds.), Proc. 1992 Les Houches Summer School, North-Holland, Amsterdam (1995).

[5] J. H. Jureit, T. Krajewski, T. Schucker and C. A. Stephan, Acta Phys. Polon. B 38 (2007) 3181 arXiv:0705.0489 [hep-th]].

[6] M. Sakellariadou, J. Phys. Conf. Ser. 442 (2013) 012015 arXiv:1301.4687 [hep-th]].

[7] K. van den Dungen and W. D. van Suijlekom, Rev. Math. Phys. 24 (2012) 1230004 arXiv:1204.0328 [hep-th]].

[8] A. Connes, JHEP 0611 (2006) 081 hep-th/0608226.

[9] A. H. Chamseddine, A. Connes and M. Marcolli, Adv. Theor. Math. Phys. 11 (2007) 991 hep-th/0610241.

[10] A. H. Chamseddine and A. Connes, Phys. Rev. Lett. 99 (2007) 191601 arXiv:0706.3690 [hep-th]].

[11] A. H. Chamseddine and A. Connes, JHEP 1209 (2012) 104 arXiv:1208.1030 [hep-ph]].

[12] C. A. Stephan, arXiv:1305.3066 [hep-ph].

[13] A. H. Chamseddine and A. Connes, Fortsch. Phys. 58 (2010) 553 arXiv:1004.0464 [hep-th]].

[14] A. Devastato, F. Lizzi and P. Martinetti, JHEP 1401 (2014) 042 arXiv:1304.0415 [hep-th]].

[15] A. H. Chamseddine, A. Connes and W. D. van Suijlekom, J. Geom. Phys. 73 (2013) 222 arXiv:1304.7583 [math-ph]].

[16] A. H. Chamseddine, A. Connes and W. D. van Suijlekom, JHEP 1311 (2013) 132 arXiv:1304.8050 [hep-th]].

[17] S. Ishihara, H. Kataoka, A. Matsukawa, H. Sato and M. Shimojo, arXiv:1311.4944 [hep-th].

[18] W. Beenakker, W. D. van Suijlekom and T. v. d. Broek, arXiv:1409.5982 [hep-th].

[19] W. Beenakker, W. D. van Suijlekom and T. v. d. Broek, arXiv:1409.5983 [hep-th].

[20] W. Beenakker, W. D. van Suijlekom and T. v. d. Broek, arXiv:1409.5984 [hep-th].

[21] W. D. van Suijlekom, JHEP 1103 (2011) 146 arXiv:1101.4804 [math-ph]].

[22] W. D. van Suijlekom, Commun. Math. Phys. 312 (2012) 883 arXiv:1104.5199 [math-ph]].

[23] W. D. van Suijlekom, Phys. Lett. B 711 (2012) 434 arXiv:1204.4070 [hep-th]].

[24] C. Estrada and M. Marcolli, Int. J. Geom. Meth. Mod. Phys. 10 (2013) 1350036 arXiv:1208.5023 [hep-th]].

[25] W. D. van Suijlekom, Annales Henri Poincare 15 (2014) 985.

[26] W. Nelson, J. Ochoa and M. Sakellariadou, Phys. Rev. Lett. 105 (2010) 101602 [arXiv:1005.4279 [hep-th]].

[27] G. Lambiase, M. Sakellariadou and A. Stabile, JCAP 1312 (2013) 020 arXiv:1302.2336 [gr-qc]].

[28] A. H. Chamseddine, A. Connes and V. Mukhanov, arXiv:1409.2471 [hep-th].

[29] F. D'Andrea, F. Lizzi and P. Martinetti, J. Geom. Phys. 82 (2014) 18 arXiv:1305.2605 [math-ph]].
[30] S. Farnsworth and L. Boyle, arXiv:1303.1782 [hep-th].

[31] M. A. Kurkov, F. Lizzi and D. Vassilevich, Phys. Lett. B 731 (2014) 311 arXiv:1312.2235 [hep-th]].

[32] B. Iochum, C. Levy and D. Vassilevich, Commun. Math. Phys. 316 (2012) 595 arXiv:1108.3749 [hep-th]].

[33] M. Reuter and F. Saueressig, JHEP 1112 (2011) 012 arXiv:1110.5224 [hep-th]].

[34] J. Ambjørn, J. Jurkiewicz and R. Loll, Phys. Rev. Lett. 95 (2005) 171301 hep-th/0505113.

[35] T. P. Sotiriou, M. Visser and S. Weinfurtner, Phys. Rev. Lett. 107 (2011) 131303 arXiv:1105.5646 [gr-qc]].

[36] S. Carlip, arXiv:0909.3329 [gr-qc].

[37] S. Carlip, AIP Conf. Proc. 1483 (2012) 63 arXiv:1207.4503 [gr-qc]].

[38] D. Benedetti and J. Henson, Phys. Rev. D 80 (2009) 124036 arXiv:0911.0401 [hep-th]].

[39] P. Horava, Phys. Rev. Lett. 102 (2009) 161301 arXiv:0902.3657 [hep-th]].

[40] T. P. Sotiriou, M. Visser and S. Weinfurtner, Phys. Rev. D 84 (2011) 104018 arXiv:1105.6098 [hep-th]].

[41] G. Amelino-Camelia, M. Arzano, G. Gubitosi and J. Magueijo, Phys. Rev. D 88 (2013) 103524 arXiv:1309.3999 [gr-qc]].

[42] G. Amelino-Camelia, M. Arzano, G. Gubitosi and J. Magueijo, Phys. Lett. B 736 (2014) 317 arXiv:1311.3135 [gr-qc]].

[43] G. Calcagni, Phys. Lett. B 697 (2011) 251 arXiv:1012.1244 [hep-th]].

[44] G. Calcagni and G. Nardelli, Phys. Rev. D 88 (2013) 124025 arXiv:1304.2709 [math-ph]].

[45] G. Calcagni, A. Eichhorn and F. Saueressig, Phys. Rev. D 87 (2013) 12, 124028 arXiv:1304.7247[hep-th]].

[46] O. Lauscher and M. Reuter, JHEP 0510 (2005) 050 hep-th/0508202.

[47] M. Reuter and F. Saueressig, Lect. Notes Phys. 863 (2013) 185 arXiv:1205.5431 [hep-th]].

[48] S. Rechenberger and F. Saueressig, Phys. Rev. D 86 (2012) 024018 arXiv:1206.0657 [hep-th]].

[49] L. Modesto, Class. Quant. Grav. 26 (2009) 242002 arXiv:0812.2214 [gr-qc]].

[50] L. Modesto and P. Nicolini, Phys. Rev. D 81 (2010) 104040 arXiv:0912.0220 [hep-th]].

[51] E. Magliaro, C. Perini and L. Modesto, arXiv:0911.0437 [gr-qc].

[52] F. Caravelli and L. Modesto, arXiv:0905.2170 [gr-qc].

[53] L. Modesto, arXiv:0905.1665 [gr-qc].

[54] G. Calcagni, D. Oriti and J. Thürigen, Class. Quant. Grav. 31 (2014) 135014 arXiv:1311.3340 [hep-th]].

[55] A. Eichhorn and S. Mizera, Class. Quant. Grav. 31 (2014) 125007 arXiv:1311.2530 [gr-qc]].

[56] D. Benedetti, Phys. Rev. Lett. 102 (2009) 111303 arXiv:0811.1396 [hep-th]].

[57] M. Arzano and T. Trzesniewski, Phys. Rev. D 89 (2014) 124024 arXiv:1404.4762 [hep-th]].

[58] E. Alesci and M. Arzano, Phys. Lett. B 707, 272 (2012) arXiv:1108.1507 [gr-qc]].

[59] A. Codello, R. Percacci and C. Rahmede, Annals Phys. 324 (2009) 414 arXiv:0805.2909 [hep-th]].

[60] A. O. Barvinsky and G. A. Vilkovisky, Nucl. Phys. B 282 (1987) 163. 
[61] A. O. Barvinsky and G. A. Vilkovisky, Nucl. Phys. B 333 (1990) 471.

[62] A. Codello and O. Zanusso, J. Math. Phys. 54 (2013) 013513 arXiv:1203.2034 [math-ph]].

[63] D. V. Vassilevich, Phys. Rept. 388 (2003) 279 hep-th/0306138.
[64] A. Devastato, Phys. Lett. B 730 (2014) 36 arXiv:1309.5973 [hep-th]].

[65] G. Calcagni, L. Modesto and G. Nardelli, arXiv:1408.0199 [hep-th].

[66] S. Carlip, R. A. Mosna and J. P. M. Pitelli, Phys. Rev. Lett. 107, 021303 (2011) arXiv:1103.5993 [gr-qc]]. 